\title{
Structure and magnetization of two-dimensional vortex arrays in the presence of periodic pinning
}

\author{
Toby Joseph* and Chandan Dasgupta ${ }^{\dagger}$ \\ Centre for Condensed Matter Theory, Department of Physics, Indian Institute of Science, Bangalore 560012, India \\ and Condensed Matter Theory Unit, JNCASR, Bangalore 560064, India
}

\begin{abstract}
Ground-state properties of a two-dimensional system of superconducting vortices in the presence of a periodic array of strong pinning centers are studied analytically and numerically. The ground states of the vortex system at different filling ratios are obtained using a simple geometric argument under the assumption that the penetration depth is much smaller than the spacing of the pin lattice. The results of this calculation are confirmed by numerical studies in which simulated annealing is used to locate the ground states of the vortex system. The zero-temperature equilibrium magnetization as a function of the applied field is obtained by numerically calculating the energy of the ground state for a large number of closely spaced filling ratios. The results show interesting commensurability effects such as plateaus in the $B-H$ diagram at simple fractional filling ratios.
\end{abstract}

\section{INTRODUCTION}

In the mixed phase of type-II superconductors, magnetic flux penetrates the sample in the form of quantized vortex lines. ${ }^{1}$ The amount of flux carried by each vortex line is equal to the basic flux quantum $\Phi_{0}=h c /(2 e)=2.07$ $\times 10^{-7} \mathrm{G} \mathrm{cm}^{2}$. These vortex lines form a special physical system known as "vortex matter." In the absence of any pinning sites in the material, the vortex lines form a triangular lattice known as the Abrikosov lattice. ${ }^{2}$

Equilibrium and transport properties of the mixed phase of type-II superconductors are strongly affected by the presence of pinning centers, either intrinsic to the system or artificially generated. Understanding the effects of pinning in these systems is very important for practical applications because the presence of pinning strongly influences the value of the critical current in the mixed phase.

In recent years, a variety of nanofabrication techniques have been used to create periodic arrays of pinning centers in thin-film superconductors. ${ }^{3-15}$ Such arrays may consist of microholes ("antidots"), ${ }^{3-8}$ defects produced by the bombardment of ion ${ }^{9}$ or electron ${ }^{10}$ beams, or magnetic dots. ${ }^{11-15}$ These pinning centers are "strong" in the sense that each pinning site can trap one or more vortices at low temperatures. The effects of periodic pinning depend strongly on the relative values of $B_{\phi}$ and $B$, where $B_{\phi}=\rho_{p} \Phi_{0}\left(\rho_{p}\right.$ is the areal density of the pinning centers) is the so-called "matching field," and $B$ is the magnetic induction that determines the areal density $\rho_{0}$ of vortices $\left(\rho_{0}=B / \Phi_{0}\right)$. The filling ratio, $n$, defined as $n \equiv B / B_{\phi}$, measures the commensurability of the vortex system with the underlying pin lattice. The interplay between the lattice constant of the pin array (determined by $B_{\phi}$ ) and the intervortex separation (determined by $B$ ) can lead to a variety of interesting effects in such systems.

Some of these effects have been observed in recent experiments. Imaging experiments using various techniques such as Bitter decoration, ${ }^{5}$ Lorentz microscopy, ${ }^{9}$ and scanning Hall-probe microscopy ${ }^{6,8}$ have shown the formation of ordered structures of the vortex system at low temperatures for commensurate values of $n$. Magnetization measurements ${ }^{3,4,7}$ in the irreversible (vortex solid) regime have demonstrated the occurrence of anomalies at certain harmonics of $B_{\phi}$. The effectiveness of pinning at integral values of $n$ has been found ${ }^{7,11-15}$ to produce regularly spaced sharp minima in the resistivity versus field curve. A pinninginduced reconfiguration of the vortex lattice has been observed $^{14}$ in a thin-film superconductor with a rectangular array of magnetic dots. Some of these effects have been studied theoretically, using analytic ${ }^{16}$ and numerical ${ }^{17-22}$ methods. Experimental realizations of a system of interacting "particles" in the presence of an external periodic potential are also obtained in colloidal suspensions in interfering laser fields, ${ }^{23}$ and in periodic arrays of optical traps. ${ }^{24}$

In this paper, we have used analytic and numerical methods to analyze the zero-temperature structure of vortex arrays in the presence of periodic pinning. We have also carried out a numerical study of the zero-temperature equilibrium magnetization of a superconducting film with a square array of pinning centers as a function of the applied field. In Sec. II, we consider the ground states of a vortex system in a square array of pinning centers for fillings less than unity. We look at a class of structures that are Bravais lattices with one vortex per basis if the filling $n$ is of the form $1 / q$, and with $p$ vortices per basis if $n=p / q$ ( $p$ and $q$ are integers greater than unity, with $p<q$ ). The structure with the lowest energy in this class can be obtained rather easily. We find that the "ground-state" structure obtained this way matches those obtained from experiments ${ }^{9}$ and simulations ${ }^{17,20}$ for a large number of simple fractional values of $n$. The results obtained in this section can also be used to predict the ground-state structures for $1<n<2$. In Sec. III, we consider the groundstate structures for fillings greater than 2 . In these calculations, we use simple geometric arguments to arrive at the ground states. This analysis is performed under the assumption that the range of the intervortex interaction, which is set by the penetration depth, is much smaller than the spacing between the pinning sites. We show that the ground-state structures obtained from this simple analysis match the ones obtained from simulated annealing. This analysis is extended 
to rectangular and triangular pin lattices in Sec. IV. In Sec. V, the zero-temperature equilibrium magnetization of a vortex system in a square array of pinning centers is obtained by first calculating the ground-state energy as a function of the magnetic induction and then finding the applied field from numerical differentiation of the data. The ground-state energies for different values of the magnetic induction are obtained using a simulated annealing procedure. The calculated $B-H$ curve exhibits interesting commensurability effects, manifested as plateaus occurring at simple rational values of the filling fraction $n$. The main results of our study are summarized in Sec. VI.

\section{GROUND STATES FOR A SQUARE PIN ARRAY WITH FILLING RATIO LESS THAN 1}

We consider a superconducting film that has a square array of pinning sites with lattice constant $d$. The magnetic field is assumed to be perpendicular to the surface of the film. The "matching field" $B_{\Phi}$ is then given by $B_{\phi}$ $\equiv \Phi_{0} / d^{2}$, and the filling fraction $n$ is given by $n=B / B_{\phi}$ $=B d^{2} / \Phi_{0}$ where $B$ is the magnetic induction. We assume that the pinning potential is much stronger than the intervortex interaction, but is of extremely short range. The large strength of the pinning potential implies that the vortices must occupy pinning sites as long as the number of vortices does not exceed the number of pinning sites. We also assume that a pinning site cannot accommodate more than one vortex. If the pinning centers in the film are microholes, then this assumption amounts to the requirement ${ }^{25}$ that the radius of each hole is close to two times the coherence length $\xi$ of the superconductor. These assumptions ensure that interstitial vortices appear only when the filling fraction $n$ is greater than unity. The assumed short range of the pinning potential can be justified if the defect diameter is small compared to the defect spacing $d$. Another assumption that we make in most of our calculations is that the intervortex interaction falls off rapidly with distance. This is ensured if the penetration depth $\lambda$ is much smaller that the pin-lattice spacing $d$. In our calculations, we take the ratio $\lambda / d$ to be 10 . This value is appropriate for the pin lattice of Ref. 9. We consider temperatures that are low enough to neglect effects of depinning and vortex-lattice melting. The problem of finding the structure of the vortex system then reduces to locating the ground state in the presence of the pinning potential.

Consider now fillings of the form $n=1 / q, q$ being an integer greater than 1. Let us look at Bravais lattices that can be formed for a specific $n$ by distributing the vortices on the square pin lattice with one vortex per basis. The motivation for considering such lattices is that this will automatically ensure that there is no shear of the vortices with respect to the pin lattice, since the forces on a vortex due to other vortices will add up exactly to zero. The unit-cell area of these structures has to be $q . d^{2}$. So the possible unit cells can be obtained by factorizing $q$ into products of the form $r$.s $(r$ and $s$ are integers), arranging the vortices at the corners of rectangles of dimension $r d \times s d$, and then sliding the parallel sides relative to each other. This procedure produces a large number of structures depending on the value of $n$ and we (a)

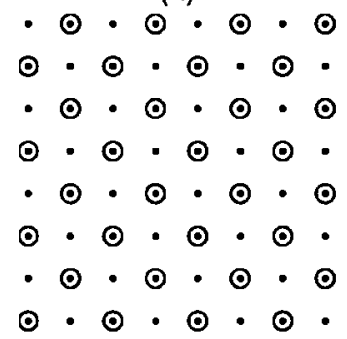

(b)

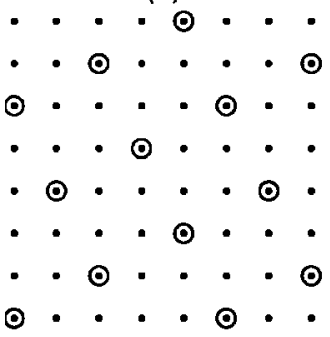

(c)

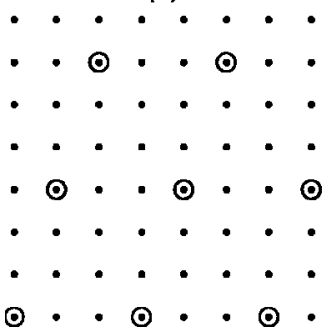

(d)

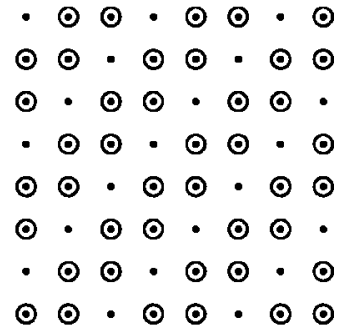

(e)

$\circ \cdot 0 \% \cdot 0 \cdot 0$

- 0 - 0 - 00

$\circ 0 \cdot 0 \cdot 0 \%$.

$0 \cdot 0 \cdot 0 \%$

- 0 - 00 - 0 .

$0 \cdot 0 \% \cdot 0 \cdot 0$

- 0 - $0 \cdot 0 \%$

$\circ \odot \cdot 0 \cdot 0 \%$.

(f)

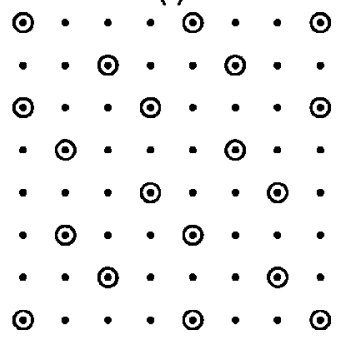

FIG. 1. The ground-state structures for a few filling fractions $n<1$. The different filling fractions are (a) $1 / 2$, (b) $1 / 5$, (c) $1 / 9$, (d) $2 / 3$, (e) $3 / 5$, and (f) $2 / 7$. The dots in the figures represent the pinning sites and the circles represent the vortices.

have to pick the one that minimizes the energy. For small values of $q$, this can be done by hand, but as $q$ becomes large and highly factorizable, the number of possible structures increases rapidly. In such cases, we have resorted to computers to generate these structures and compare their energies.

The structures so obtained for fillings $1 / 2$ and $1 / 4$ match those found in the imaging experiment. ${ }^{9}$ Also for fillings $1 / 2$, $1 / 3,1 / 4,1 / 5,1 / 8,1 / 9,1 / 10$, and $1 / 15$, we find the same structures as those obtained by solving the "greedy latticegas model" 26 exactly. This is understandable because when the intervortex interaction falls off rapidly as the distance is increased above the defect spacing $d$, the ground state can be attained by finding the lattice that maximizes the shortest distance between vortex pairs. If two structures have the same value and number of shortest distances, then the next shortest distance should be maximized, and so on. For fillings $1 / 2,1 / 3,1 / 4$, and $1 / 5$, our analysis also yields the same structures as those found in the large $U_{e}\left(U_{e}\right.$ is the energy of on-site repulsion between two electrons) limit of the neutral Falicov-Kimball model. ${ }^{27}$ In Figs. 1(a)-1(c), we show the structures so obtained for a few fillings of the form $n$ $=1 / q$. The ground-state structure shown in Fig. 1(b) for $n$ $=1 / 5$ is different from that found in Ref. 18 from a simulated annealing calculation. This difference is probably due to the 
use of a different (logarithmic) intervortex potential in Ref. 18.

Let us now compare the energies of the nearest and nextnearest neighbors in one of these lattices. The interaction energy between two vortices separated by a distance $r$ is given by the expression

$$
U(r)=\frac{\Phi_{0}^{2} t}{8 \pi^{2} \lambda^{2}} K_{0}\left(\frac{r}{\lambda}\right),
$$

where $K_{0}$ is the zeroth-order Hankel function of imaginary $\operatorname{argument}$ and $t$ is the film thickness. For $n=1 / 2$, the nearestneighbor distance is $\sqrt{2} d$ and the next-nearest-neighbor distance is $2 d$. So the interaction energies are, to within a constant prefactor, given by

$$
\begin{aligned}
& U_{n} \propto K_{0}\left(\frac{\sqrt{2} d}{\lambda}\right)=0.2 \times 10^{-6}, \\
& U_{n n} \propto K_{0}\left(\frac{2 d}{\lambda}\right)=0.6 \times 10^{-10} .
\end{aligned}
$$

One can see here that there is an orders-of-magnitude difference in these energies which cannot be compensated by differences arising from interactions with more distant neighbors. This difference is going to be more prominent at lower densities. This tells us that the maximization of the shortest intervortex distance in a lattice for a given filling would lead to the ground states, provided the lattice spacing is large compared to the penetration depth of the film. This, in fact, is exactly the definition of the "greedy lattice gas." However, one has to be cautious about this method because, as noted in Ref. 26, the structures can be strongly dependent on the form of the potential in certain ranges of $n$ and we can even have aperiodic structures as ground states. The ground-state structures shown here have been cross checked with simulations to ensure that they are indeed the lowest-energy configurations. To give an example of a case in which this treatment does not lead to the true ground state, we found that for filling $1 / 16$, the energy per vortex for the structure with minimum energy obtained this way was greater than that for filling $1 / 15$, implying that the structure obtained for $n=1 / 16$ was not the ground state.

When the filling fraction is of the form $p / q$ with $p$ not equal to 1, one can look for ground states in a subset of structures where the unit cell has the size $q d^{2}$ with $p$ vortices in a basis. We have shown in Figs. 1(d)-1(f) some of the ground-state structures obtained this way. These structures match those obtained from our simulated annealing calculation. These ground states show the "stripe" structure predicted by Watson ${ }^{26}$ and Kennedy ${ }^{27}$ in appropriate density ranges.

\section{GROUND STATES FOR A SQUARE PIN ARRAY WITH FILLING RATIO GREATER THAN 2}

If $n$ is greater that 1 but smaller than 2, then the groundstate structures are similar to the ones for the case of $n$ less than 1 . The only difference is that the pinning sites are all

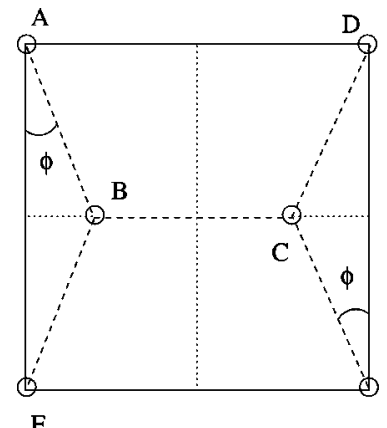

(a)

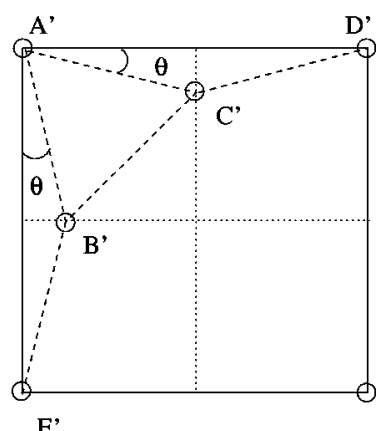

(b)
FIG. 2. Putting two vortices in a square. The configuration in (a) is the global energy minimum, and the configuration in (b) can at best be a local minimum of the energy. Angles are $\theta=15^{\circ}$ and $\phi$ $=24.49^{\circ}$. The square is drawn for easy visualization and the dotted lines are the bisectors of the sides. Note that there is already one pinned vortex at each of the corners of the square. Distances are $A B=B C=B E=D_{m a}$ and $A^{\prime} B^{\prime}=B^{\prime} C^{\prime}=B^{\prime} E^{\prime}=D_{m b}$.

occupied and the centers of the square unit cells of the pin lattice now act as new pinning centers for interstitial vortices. But things look different when the filling goes above the value $n=2$. For such values of $n$, we can no longer place the interstitial vortices at the centers of the squares and look for simple structures obtained this way. Also, we now have to start looking into the stability of the structures since the square symmetry would not be present.

\section{A. The ground state for $n=5 / 2$}

Here we are faced with the task of placing more than one vortex in a square. Before going to the problem of finding the ground state for $n=5 / 2$, let us ask a more basic question: given a single unit cell of the square pin lattice with each corner occupied by a vortex, how can we arrange two more vortices inside this square so as to minimize the energy? Since "greedy lattice gas" has been a good approximation for the previous cases, we try to tackle this problem by using the "maximizing the shortest distance" method. In order to stabilize an interstitial vortex by maximizing the shortest distance, its distance from at least three nearest vortices must be the shortest distance. It is also required that these vortices must be spread in such a way that if we draw straight lines from the vortex in question to these neighbors, the angles formed by adjacent lines must be less than $180^{\circ}$. The proof of this statement is given in the Appendix.

It can be seen from the symmetry of the problem that we have to place the two vortices on the lines joining the centers of the sides to meet the condition mentioned above. This leaves us with only two possible ways of doing it, which are shown in Fig. 2. In Fig. 2(a) the shortest distance $D_{m a}$ can be obtained by solving the equation

$$
\frac{1}{4}\left(d-D_{m a}\right)^{2}+\frac{d^{2}}{4}=D_{m a}^{2} .
$$

On solving this equation, we get $A B=B C=B E=D_{m a}$ $=(\sqrt{7}-1) d / 3$. In Fig. 2(b) the vortices $A^{\prime}, B^{\prime}$, and $C^{\prime}$ form 


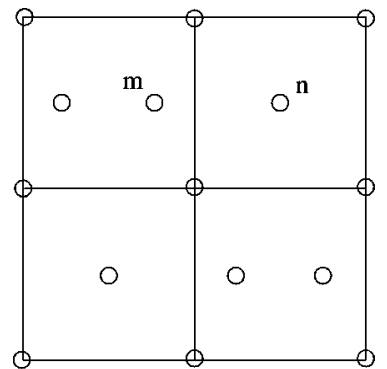

(a)

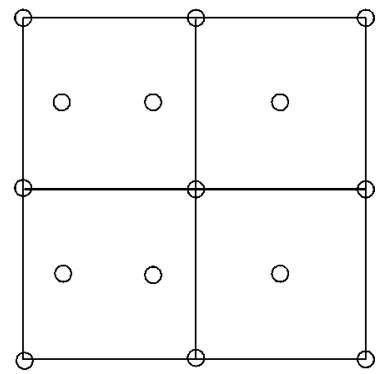

(c)

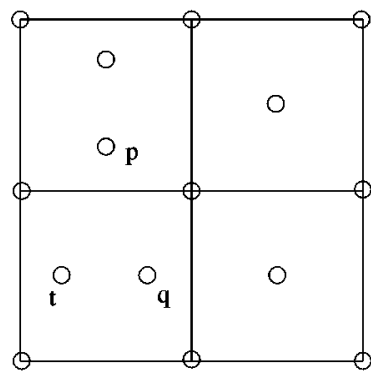

(b)

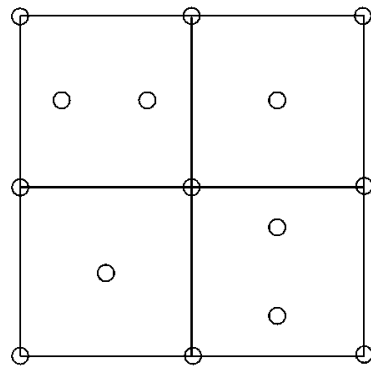

(d)
FIG. 3. Possible $2 d \times 2 d$ unit cells for $n=5 / 2$. The unit cell (a) is the lowest-energy configuration for $d / \lambda=10$. For much larger values of $d / \lambda$, the unit cell with the lowest energy will be the one in (b).

an equilateral triangle. Thus the nearest-neighbor distance $A^{\prime} B^{\prime}=B^{\prime} C^{\prime}=B^{\prime} E^{\prime}$ is

$$
D_{m b}=\sec \left(15^{\circ}\right) \frac{d}{2} .
$$

The angle $\phi$ in Fig. 2(a) is $24.49^{\circ}$, and the angle $\theta$ in Fig. 2(b) is $15^{\circ}$. The interaction energies corresponding to these two distances for $d / \lambda=10$ are

$$
\begin{aligned}
U_{A B} \propto K_{0}\left(\frac{\sqrt{7}-1}{3 \lambda} d\right) & \simeq 2.2 \times 10^{-3}, \\
U_{A^{\prime} B^{\prime}} \propto K_{0}\left(\frac{\sec \left(15^{\circ}\right)}{2 \lambda} d\right) & \simeq 3.0 \times 10^{-3} .
\end{aligned}
$$

From comparing these two energies it is clear that Fig. 2(a) is the global minimum, whereas the configuration of Fig. 2(b) can at best be a local minimum.

Coming back to the $n=5 / 2$ case, we now have to build up the lattice with an equal number of two types of squaresone with two interstitial vortices and the other with one interstitial vortex. Note that here we have neglected structures that have three or more interstitial vortices inside a square unit cell because such structures would drastically bring down the nearest-neighbor distance. Let us now look at the possible units cells of size $2 d \times 2 d$ that can be made out of these two types of squares. These are shown in Fig. 3. If one constructs the lattice with these unit cells, the configuration of Fig. 3(b) offers the least number of next-nearest neighbors, the number of nearest neighbors being the same in all the cases. So one can expect Fig. 3(b) to be the ground-state unit cell, at least for large values of $d / \lambda$. However, the unit cell of Fig. 3(a) is preferred if $d / \lambda$ is not very large. This can be understood in the following way: the advantage that Fig. 3(b) has over Fig. 3(a) is that it has only half the number of next-nearest neighbors (interactions like that between vortices $m$ and $n$ ) compared to that of Fig. 3(a). But this is done at the cost of bringing in interactions such as those between vortex pairs $(p, q)$ and $(p, t)$ for every "gain" of a nextnearest-neighbor interaction. The energies of these two interactions for $d / \lambda=10$ are found to be quite close. These energies are

$$
\begin{aligned}
& U_{m n} \propto K_{0}\left(r_{m n} / \lambda\right) \simeq 3.2 \times 10^{-4}, \\
& U_{p q} \propto K_{0}\left(r_{p q} / \lambda\right) \simeq 1.9 \times 10^{-4},
\end{aligned}
$$

where $r_{m n}$ and $r_{p q}$ are the distances between vortices $m$ and $n$, and $p$ and $q$ in Fig. 3, respectively. It is clear from this comparison that the unit cell of Fig. 3(a) would be preferred for $d / \lambda=10$.

The ratio of interaction energies of the next-nearest and the nearest neighbors is 0.07 for this lattice when $d / \lambda=10$. This energy difference is appreciable here, so that we can expect that the unit cell at which we arrived is the correct one. Note that any net force that might be present on one of the interstitial vortices due to the asymmetry in the structure can be compensated by extremely small displacements from the positions obtained from the "maximization of the shortest distance" method.

\section{B. The ground state for $n=3$}

When the filling fraction equals 3 , we have to build up the lattice using blocks of the type of Fig. 2(a). Again, looking at unit cells of size $2 d \times 2 d$ or smaller, we have the configurations shown in Fig. 4 to consider. Here it is easy to see that the unit cell of Fig. 4(b) is preferred over the others. This is because it is the configuration that maximizes the minimum distance between any two vortices in different squares, the distances between vortices within one square being the same in all the configurations. Again comparing the nearest interaction and the next-nearest one, we have

$$
\begin{aligned}
U_{n} \propto K_{0}\left(r_{s p} / \lambda\right) & \simeq 2.2 \times 10^{-3}, \\
U_{n n} \propto K_{0}\left(r_{p q} / \lambda\right) & \simeq 1.9 \times 10^{-4} .
\end{aligned}
$$

There is an appreciable difference between these two values, and hence, the ground state at which we have arrived is reasonable. When the filling lies between 2 and 3, one can safely assume that the ground-state structure can be built up using squares of the type of Fig. 2(a), and squares that have one vortex at the center. In fact we make use of this in our simulations to arrive at the ground states, as described in Sec. V.

\section{The ground state for $n=4$}

Here we have to place three interstitial vortices in one square. This is nontrivial since even if we ensure that the shortest distance is maximized in one square, two vortices in 


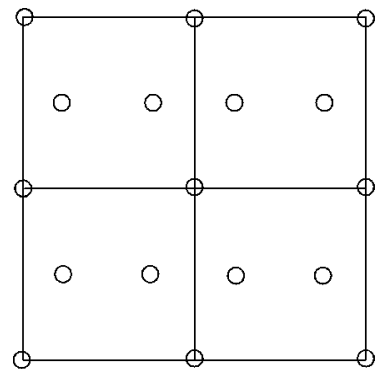

(a)

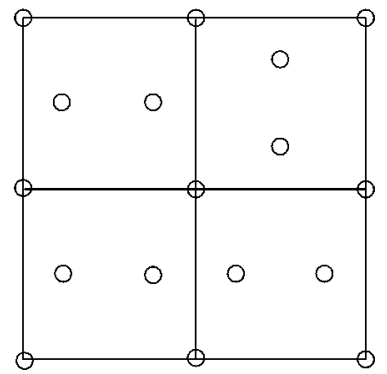

(c)

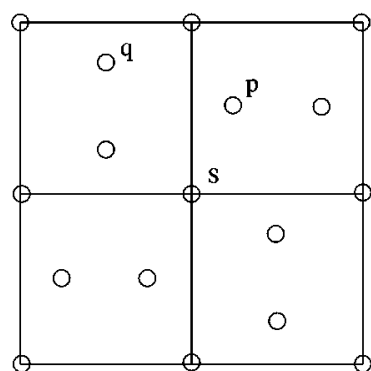

(b)

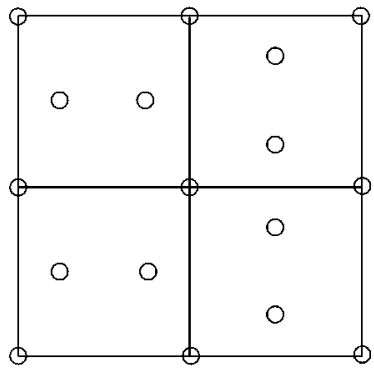

(d)
FIG. 4. Possible $2 d \times 2 d$ unit cells for $n=3$. Configuration (b) can be easily seen to offer the maximum next-nearest-neighbor distance, the nearest-neighbor distances being the same and equal in number in each case.

nearby squares may be closer to each other than the shortest distance within a square when we create the lattice. Note that we did not come across this problem in the $n=5 / 2$ or $n=3$ fillings. To illustrate this problem, we show in Fig. 5(a) a single cell of a square lattice with three interstitial vortices, which may very well be a local minimum configuration. But if we try to build the lattice using this cell, we cannot do it without bringing the vortices in nearby squares closer than the minimum distance in an individual cell. So what we need to do is to look for a pattern that will include vortices in different squares while doing the minimization of the shortest distance. We can solve this problem trigonometrically. Consider Fig. 6. The solution that we are looking for can be

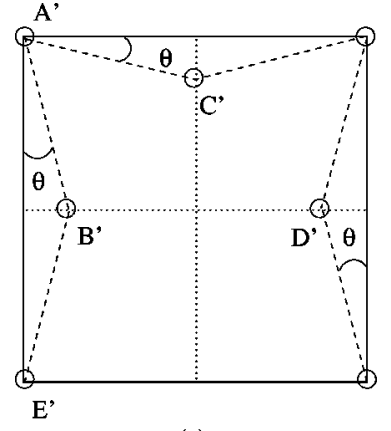

(a)

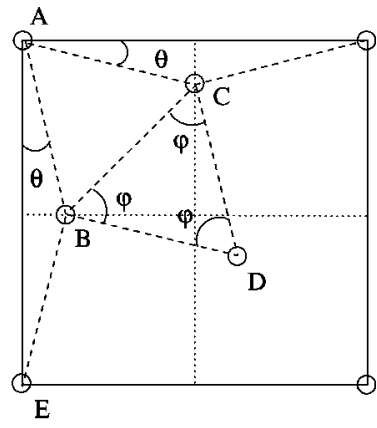

(b)
FIG. 5. Two possible ways of arranging three vortices in a square so as to maximize the shortest distance. The configuration in (a) offers the best arrangement if looked in isolation, but the configuration in (b) wins out when one has to construct a lattice of the unit cells. The angles $\theta=15^{\circ}$ and $\varphi=60^{\circ}$.

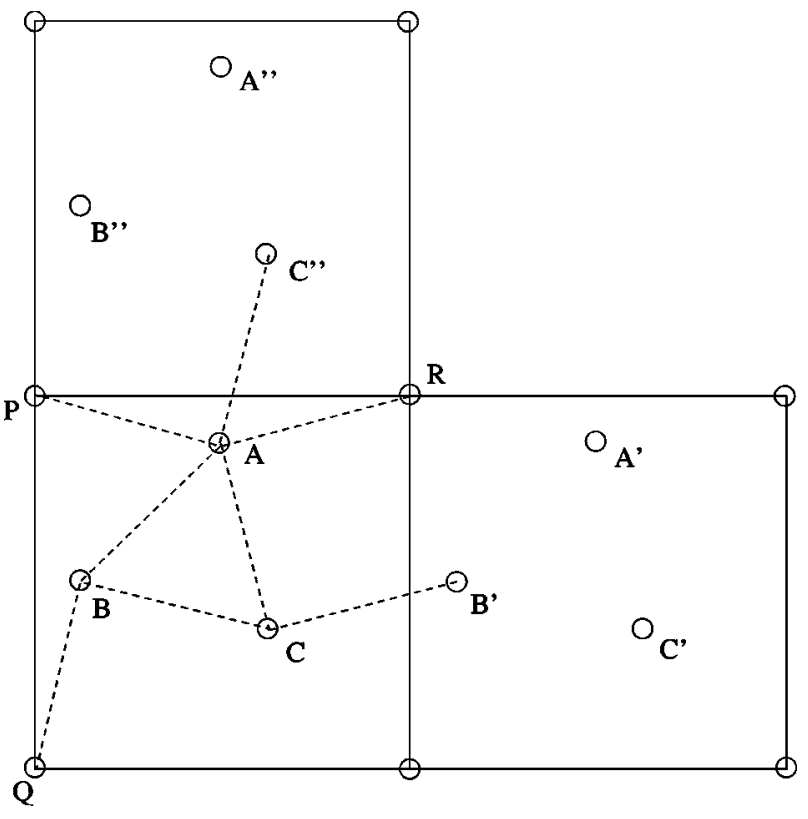

FIG. 6. The distances one has to equate when maximizing the shortest distance for $n=4$, taking into consideration distances between vortices in neighboring squares. Note that the unit-cell size here is one square unit.

obtained by solving the equations

$$
\begin{gathered}
A B=B C=C A=D_{s}, \\
C B^{\prime}=A C^{\prime \prime}=A P=D_{s} .
\end{gathered}
$$

Note that here we have assumed a unit-cell size $d \times d$. On solving these equations, we obtain the unit cell shown in Fig. $5(\mathrm{~b})$. In the figure, the angle $\theta$ equals $15^{\circ}$ and the angle $\varphi$ is $60^{\circ}$. In this lattice, the shortest distance is $D_{s}$ $=\sec \left(15^{\circ}\right) d / 2$ and the next shortest distance is $D_{n s}$ $=3 d \sec \left(15^{\circ}\right) /(4 \sqrt{2})$.

This simple solution may not be the correct one if the lattice spacing is not large enough. For example, if $d / \lambda$ $=10$, as we have been assuming when comparing energies, then the nearest- and next-nearest-neighbor interaction energies turn out to be really close. Hence we cannot rule out the possibility of the lattice arranging in such a way that the shortest distance is reduced so as to decrease the number of nearest or next-nearest neighbors. The relevant energies for $d / \lambda=10$ are

$$
\begin{aligned}
K_{0}\left(\frac{D_{s}}{\lambda}\right) & \simeq 3.1 \times 10^{-3}, \\
K_{0}\left(\frac{D_{n s}}{\lambda}\right) & \simeq 2.1 \times 10^{-3} .
\end{aligned}
$$

So the ground state obtained above is guaranteed to be the correct one only for much larger values of $d / \lambda$. The ground states we have obtained for $n=5 / 2$ and $n=3$ match with the images from experiments ${ }^{9}$ and also the results of simulations. ${ }^{17}$ But for $n=4$, the structures found in experiments and simulations are different from the one shown in 


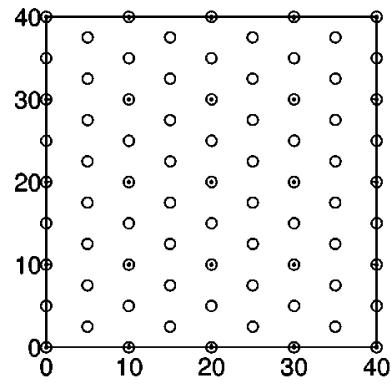

(a)

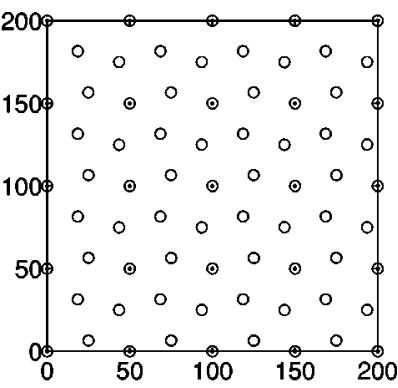

(b)
FIG. 7. The ground state for $n=4$, obtained by simulated annealing when the ratios of the penetration depth to the pin-lattice spacing are (a) 10 and (b) 50. The dark dots denote the pinning sites and the axis labels are in units of the penetration depth.

Figs. 5(b) and 6. This is expected, since in the experiment the value of the ratio $d / \lambda$ was close to 10 . Our simulation with $d / \lambda=10$ gives the ground-state structure shown in Fig. 7(a), which is similar to the one obtained in experiment. ${ }^{9}$ In our simulations with very large values of $d / \lambda$, we obtain structures similar to that in Fig. 6. The simulation result for $d / \lambda$ $=50$ is shown in Fig. 7(b), which matches well with the predicted structure. It is to be noted that the simulation result was obtained by starting the system near the expected ground state. So the claim is that it offers at least a local minimum of the interaction energy. The simulations were carried out for different system sizes from $2 d \times 2 d$ to $10 d \times 10 d$ to rule out any dependence of the results on the boundary condition.

\section{GROUND STATES FOR RECTANGULAR AND TRIANGULAR PIN ARRAYS}

One can extend this type of analysis to pinning arrays with other symmetries for finding the least energy structures for simple filling fractions. Let us first consider the case of a rectangular array of pinning sites with a pinning unit cell of dimensions $l \times b$, where we take $l$ to be the longer side of the rectangle. We shall consider here only the cases in which the filling is greater than 1 . In the absence of square symmetry, it is obvious that the ground-state structure will depend not only on the penetration depth $\lambda$, but also on the ratio $l / b$. In the following analysis, we shall always assume that $\lambda$ is much smaller than $b$, the shorter side of the basic rectangular pinning cell. When the filling is 2 , for values of $l / b$ less than $\sqrt{3}$, the ground state is one in which each interstitial vortex is at the center of the rectangle, since this ensures that the shortest distance is maximized [see Fig. 8(b)]. But when the aspect ratio exceeds $\sqrt{3}$ and the interstitial vortices are placed at the centers of the rectangles, the distance between two interstitial vortices in neighboring cells would be shorter than that between an interstitial vortex and the closest pinned vortex. This would lead to a displacement of the interstitial vortices sideways from the center, along the bisector of the shorter sides of the rectangle, to maximize the shortest distance. The resulting structure is shown in Fig. 8(a). The displacement of the vortex from the center is given by

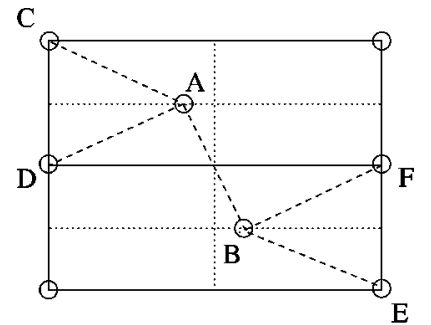

(a)

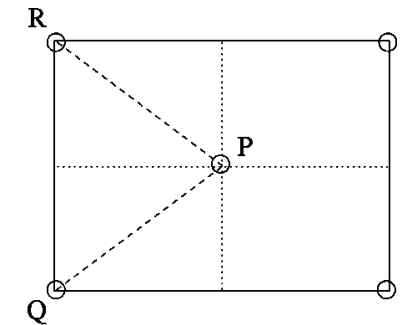

(b)
FIG. 8. The unit cells of the ground state, obtained by maximizing the shortest distance, when the filling is 2 for a rectangular array of pinning sites. (a) The unit cell when the aspect ratio is greater than $\sqrt{3}$. The interstitial vortex is displaced horizontally from the center of the rectangle by distance $D_{s}$. In the figure, distances are $A B=A C=A D=B F=B E$. (b) The ground-state unit cell when the aspect ratio is less than $\sqrt{3}$. The interstitial vortex is at the center of the rectangle.

$$
D_{s}=\frac{-l+\sqrt{4 l^{2}-9 b^{2}}}{6} .
$$

It is worth noting that since the vortex in the center would be moving towards two of the pinned vortices, and away from only one interstitial vortex per unit cell, the displacement will approach the value given above only when the ratio $l / b$ is appreciably large and in the limit of small penetration depth compared to the sides of the rectangle. For example, we have found in our simulations that even when the ratio $l / b$ is 2 , the ground state for $b / \lambda=15$ is one in which the interstitial vortex is very close to the center, whereas for $l / b=3$ and $b / \lambda=15$, the ground-state structure is quite close to the one obtained from maximizing the shortest distance. Also, if the ratio $l / b$ becomes too large, the analysis will have to include more than two of the interstitial vortices, since now the solution such as that shown in Fig. 8(a) can lead to two vortices being closer in the next-nearest cells or ones even further apart.

In trying to arrive at the lowest-energy structures for fillings $5 / 2$ and 3 , it is important to determine how one can accommodate two vortices in a rectangular cell with the shortest distance being maximized. There are two possible minima that one has to consider: one in which the vortices are arranged along the line dividing the shorter sides, and one in which they are arranged along the line dividing the longer sides, as shown in Figs. 9(a) and 9(b), respectively. The shortest distance in each case is given by

$$
\begin{aligned}
& D_{s 1}=\frac{\sqrt{4 l^{2}+3 b^{2}}-l}{3}, \\
& D_{s 2}=\frac{\sqrt{4 b^{2}+3 l^{2}}-b}{3} .
\end{aligned}
$$

If one considers the distances within the cell, the configuration of Fig. 9(b) gives the lowest energy. But for large values of the ratio $l / b$, this configuration is disfavored since it allows the vortices in one rectangle to get close to those in a neighboring one. Also, for $l / b>2$, the interstitial vortices "spill over" into the next cell, since the distance $D_{s 2}$ be- 


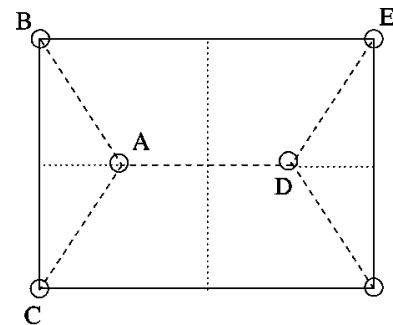

(a)

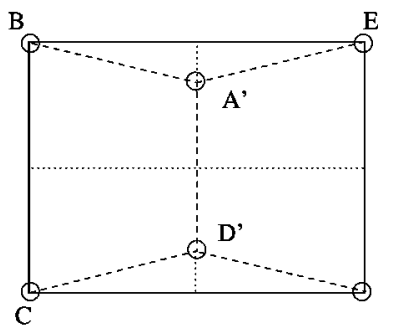

(b)
FIG. 9. The two possible ways in which the shortest distance can be maximized when there are two vortices in a rectangular cell. (a) In this case the vortices are placed on the line that divides the shorter sides of the rectangle. Distances are $A B=A C=A D=D E$ $=D_{s 1}$. (b) Here the vortices are placed parallel to the shorter sides and distances are $A^{\prime} B=A^{\prime} E=A^{\prime} D^{\prime}=C D^{\prime}=D_{s 2}$. For $l / b$ less than 2 , the configuration in (b) leads to a larger shortest distance (within the cell) than the one in (a). If $l / b$ is greater than 2 , then the distance $D_{s 2}$ becomes greater than $b$ and the vortices spill over into the next cell.

comes greater than $b$. So, one has to work out the structures for fillings $5 / 2$ and 3 case by case. For fillings between 2 and 3 , one has to choose the appropriate number of two-vortex rectangles of the right kind and single-vortex ones and arrange them so as to maximize the shortest distance appearing in the structure. We have looked at the $2 \times 2$ unit cells possible for filling $5 / 2$ for two values of the aspect ratio, $l / b$ $=4$ and $l / b=5 / 4$. The unit cells that provide the largest minimum distance are shown in Fig. 10. Note that when $l / b=4$, the vortices are arranged parallel to the longer side and in the other case, parallel to the shorter side. Also when $l / b=5 / 4$, the vortex in the single-vortex rectangular cell is not located at the center, but is slightly displaced sideways along the bisector of the shorter sides to facilitate the maximization of the second shortest distance involved. Groundstate structures obtained from simulations for different values of $l / b$ and large values of $b / \lambda$ are consistent with these predictions. One should again keep in mind that this sort of analysis cannot work if the aspect ratio is too large, since then the distances between vortices in next-nearest or further neighbor cells will become important.

For filling equal to 3 , the lowest-energy structures obtained by considering $2 \times 2$ unit cells for two values of the aspect ratio, 2 and 5/4, are shown in Fig. 11. Here too, for large values of the aspect ratio, the structure is composed of rectangular cells in which the interstitial vortices are aligned parallel to the longer side [Fig. 11(a)], whereas when the aspect ratio is smaller, the structure is made up of an alternating arrangement of rectangular cells of both types [Fig. 11(b)]. The simulated annealing results for similar values of the aspect ratio yield the structures obtained from the above analysis.

Ground-state structures obtained by simulated annealing for a rectangular pin array with $l / b=2$ and integral values of $n$ are reported in Ref. 19. In that study, the intervortex interaction was assumed to depend logarithmically on the intervortex distance. The ground-state structure found in Ref. 19 for $n=2$ is similar to that predicted by our analysis. However, the structure found there for $n=3$ is quite different

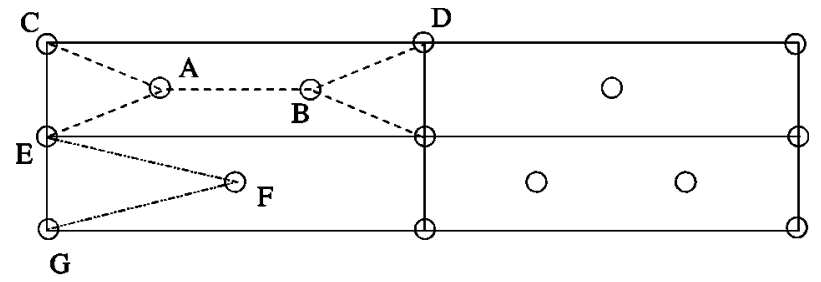

(a)

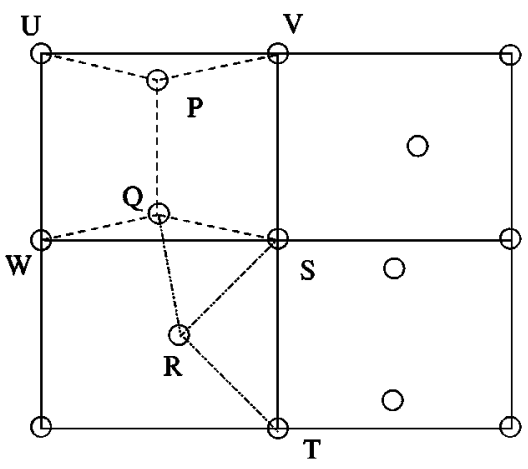

(b)

FIG. 10. The unit cells for $n=5 / 2$ for a rectangular array of pinning sites. (a) The unit cell when the aspect ratio is 4 . Distances are $A B=A C=A E=B D$. (b) The unit cell when the aspect ratio is 5/4. Here $P V=P U=P Q=Q W=Q S=D_{s 2}$ and $Q R=R S=R T$. When this structure is repeated periodically, the "image" of the vortex at $P$ would be at the same distance $Q R$ from $R$. This would ensure the stability of the vortex at $R$.

from the ones obtained from our analysis. This is another example of the importance of the detailed nature of the intervortex interaction in determining the structure of the ground state.

For a triangular array of pinning sites, it is easy to see that when the filling is greater than 1 and less than 3, the interstitial vortices will be placed on the centroids of the triangles in the limit for which one can safely apply the method of maximization of the shortest distance. So the ground states when $n$ is between 1 and 3 will be made up of parallelogram cells of the form shown in Fig. 12. These unit cells match well with the results of molecular-dynamics simulations. ${ }^{17,20}$

\section{EQUILIBRIUM MAGNETIZATION OF THE GROUND STATES}

In this section, we describe a calculation of the zerotemperature, equilibrium magnetization of a thin-film superconductor in the presence of a square array of pinning sites. The region in the $B-H$ plain in which we are interested is that just above $H_{c 1}$, when the flux tubes start entering the sample. The idea is to find the free energy $F$ of the ground state as a function of the magnetic induction $B$, and then obtain the applied magnetic field $H$ by taking a derivative of the free energy with respect to $B$. Since we are considering the zerotemperature case, the free energy is just the internal energy of the flux lattice. Since we are looking for a nearly continuous variation of the internal energy for taking the derivative, we need to locate the ground states for filling fractions sepa- 


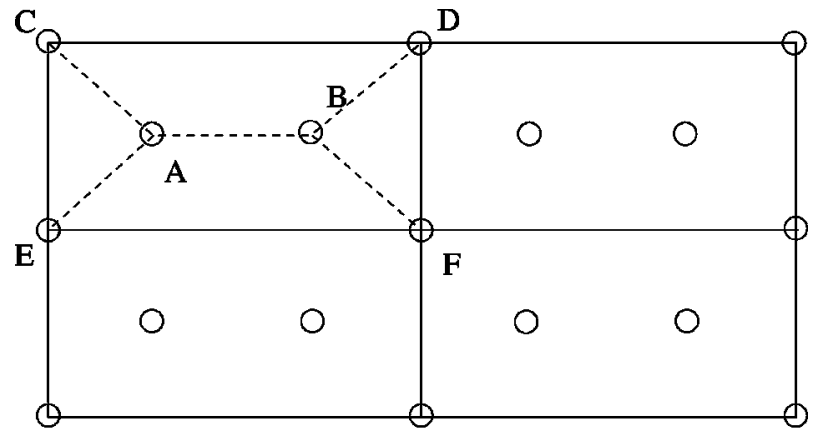

(a)

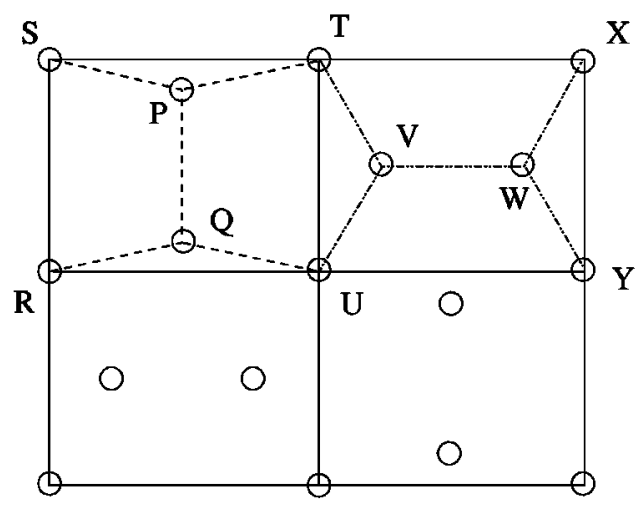

(b)

FIG. 11. Unit cells for $n=3$ for a rectangular array of pins. (a) The unit cell when the aspect ratio is 2 . Distances are $A B=A C$ $=A E=B D=B F$. (b) The unit cell for the same filling but for an aspect ratio 5/4. Here distances are $P Q=P S=P T=Q R=Q U$ $=D_{s 2}$ and $T V=U V=V W=W Y=W X=D_{s 1}$.

rated by small intervals. This would be difficult to do analytically, since the unit cells for some filling fractions can be arbitrarily large. Also, as $n$ becomes large, the simple procedure of maximization of the shortest distance is not going to yield the correct ground-state structures. So we have resorted to simulations to determine the ground states. In particular, we have used the simulated annealing technique to locate the global minima (or at least low-lying local minima close in

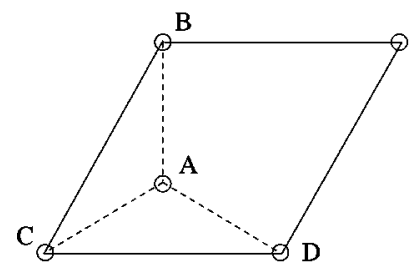

(a)

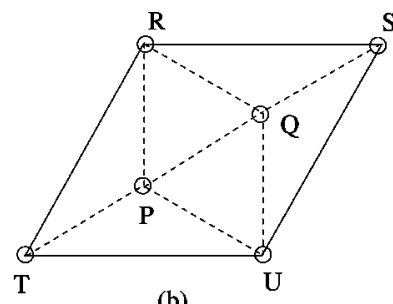

(b)
FIG. 12. Basic building blocks for generating ground states for triangular pinning arrays when the filling is between 1 and 3. (a) When there is a single interstitial vortex in a parallelogram, the shortest distance can be maximized by placing it at one of the two centroids of the triangles involved. Distances are $A B=A D=A C$ $=a / \sqrt{3}$, where $a$ is the length of the side of a pinning cell. (b) When two vortices are to be placed in a single pin cell, they have to be at the two centroids. energy to the global ones) of the part of the internal energy associated with intervortex interactions.

The Helmholtz free energy per unit volume of the superconductor at zero temperature in the presence of the pinning sites is

$$
F_{s}(n)=\frac{n \epsilon_{l}}{d^{2}}+E_{n}-\frac{n \epsilon_{p}}{d^{2}},
$$

where the first term is the line energy, the second term is the interaction energy, and the third term is the pinning energy. Here, $\epsilon_{l}$ is the line energy per unit length, $\epsilon_{p}$ is the pinning energy per unit length, and $E_{n}$ is the interaction energy per unit volume for filling fraction $n$. We note here that the pinning energy increases linearly with $n$ until $n$ becomes 1 and then remains constant, since multiple occupation of a pinning center is not allowed. Further, for simplicity, we express the pinning energy as

$$
\epsilon_{p}=m \epsilon_{l},
$$

where $m$ is a positive number whose magnitude depends on the nature of pinning. The interaction part of the free energy, $E_{n}$, is the computational input. Once we know the free energy, we can compute the applied magnetic field $H$ using the relation

$$
\frac{\partial F_{s}}{\partial B}=\frac{H}{4 \pi} .
$$

Using the standard expression ${ }^{1}$ for $\epsilon_{l}$ and taking the logarithm of the Ginzburg-Landau number to be equal to 2, we get the following expression for the applied field as a function of the filling fraction:

$$
H=\frac{\Phi_{0}}{2 \pi \lambda^{2}}\{1-m[1-\Theta(n-1)]\}+\frac{\partial E_{n}^{\prime}}{\partial n} .
$$

Here $E_{n}^{\prime}$ is given by the expression

$$
E_{n}^{\prime}=\frac{\Phi_{0}}{2 \pi \lambda^{2} N} \sum_{i>j} K_{0}\left(r_{i j} / \lambda\right),
$$

where $N$ is the number of basic pinning squares in the system and $r_{i j}$ is the separation between vortices $i$ and $j$ in the ground state for the filling fraction $n$.

The size of the systems we simulated varies from $2 d$ $\times 2 d$ to $8 d \times 8 d$. In all cases, we used periodic boundary conditions to minimize surface effects. So the minimum difference between two consecutive filling fractions was $\Delta n$ $=1 / 64$. The ratio $d / \lambda$ was taken to be 10 , as in our previous analysis. In order to save computation time, the vortices were allowed to stay only at the pinning sites when the filling was less than 1. For fillings between 1 and 2, every pinning site was occupied by a vortex which was never moved and the extra ones were allowed to move near the centers of the basic pinning squares. When the filling was greater that 2 and less than 3 , the vortex configurations were constructed using basic units of squares containing one vortex at its center and squares containing two vortices placed such that the shortest 


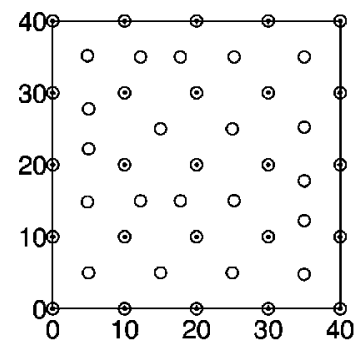

(a)

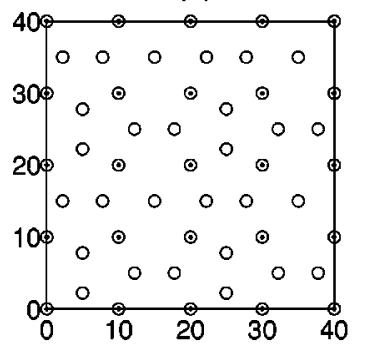

(c)

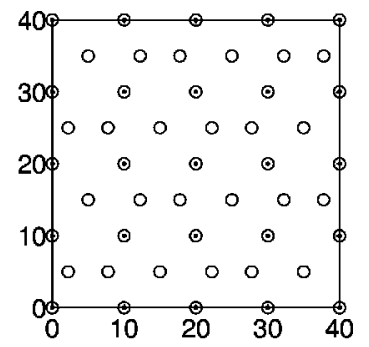

(b)

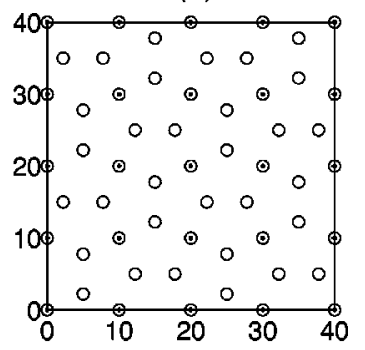

(d)
FIG. 13. The ground states for (a) $n=9 / 4$, (b) $n=5 / 2$, (c) $n$ $=11 / 4$, and (d) $n=3$ obtained by simulated annealing, as discussed in the text. The unit-cell size is $4 d \times 4 d$. The dark dots denote the pinning sites and the circles denote the vortices. The axis labels are in units of the penetration depth.

distance within a square is maximized [as in Fig. 2(a)]. These units were then moved around and twisted while cooling to arrive at the minimum-energy states. This procedure helped us to track low-lying minima faster than if we allowed vortices to move freely. Once the basic structure was thus obtained, the vortices were allowed to move freely during a second cooling schedule starting from a lower temperature to obtain the lowest-energy structure. In Fig. 13 we show some of the ground-state structures we have obtained this way for fillings between 2 and 3. For fillings $5 / 2$ and 3, we find that the structures match those obtained in experiments, ${ }^{9}$ as well as in our analysis using maximization of the shortest distance. The structures for $n=9 / 4$ and $n=11 / 4$ may not be the actual ground states, either due to the smallness of the unit cell of our simulation or the presence of many nearly degenerate local minima.

In Fig. 14 we plot the ground-state energies obtained from the simulation for different fillings. The simulation unit cell was $8 d \times 8 d$ and the energies were computed for fillings $1 / 64$ to 3 . The upper curve shows the results obtained in the presence of the pinning sites and the lower curve is the energy of the triangular lattice for the same density of vortices. Note that we have not included the pinning energy in the plot. This would bring the upper curve below the curve for the pin-free case.

From the energy versus filling fraction data, one can find the applied field using Eq. (11) and then compute the magnetization $M$ using the relation

$$
B=H+4 \pi M .
$$

In Fig. 15 we have plotted $B$ versus $H$ in the entire range of filling for which simulations were carried out, from $n=0$ to

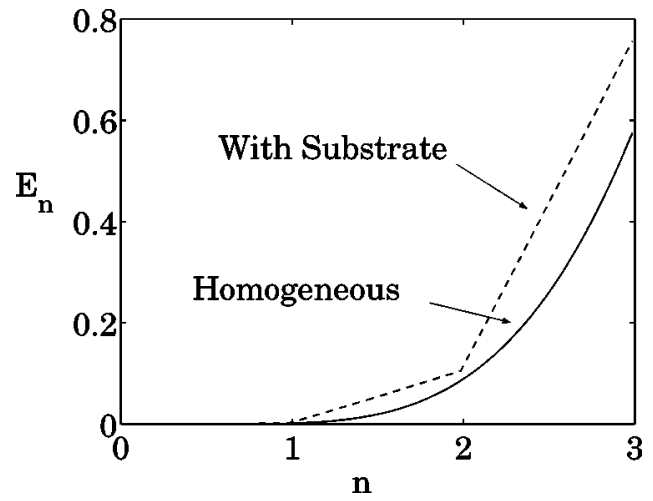

FIG. 14. The energy of the vortex lattice in the presence of a square array of pinning sites (upper dashed curve). The energy of the triangular vortex lattice in the absence of pinning is also shown for comparison (lower solid curve). The system size is $8 d \times 8 d$. The abscissa is the filling fraction $n$ and the ordinate is the total energy per unit thickness in units of $\Phi_{0}^{2} /\left(8 \pi^{2} \lambda^{2}\right)$.

$n=3$. Figures 16 and 17 show magnified versions of this plot in the regions between filling fractions 0 and 1 , and between 2 and 3, respectively. Note that we have not explicitly included the pinning energy term in our analysis. This term would just add a constant contribution to $H$ for fillings up to 1 . The features of the curve from $n=1$ to $n=2$ are the same as those in the interval between $n=0$ and $n=1$. This is due to the fact that the ground-state structures are similar in the two regions (see Sec. III). The $B-H$ plot shows flat regions at values of $B$ corresponding to fillings $1 / 8,1 / 5,1 / 4$, $1 / 2,3 / 4,4 / 5,7 / 8,1,9 / 8,6 / 5,5 / 4,3 / 2,7 / 4,9 / 5$, and 2 in the filling fraction range between 0 and 2. Also, in the range of $n$ between 2 and 3, there are roughly two plateaus, appearing near $n=2.3$ and $n=2.6$.

The observed values of the filling fractions between $n$ $=0$ and $n=1$ at which the plateaus occur indicate that these values of $n$ correspond to fillings for which the introduction of a new vortex into the system leads to the appearance of a

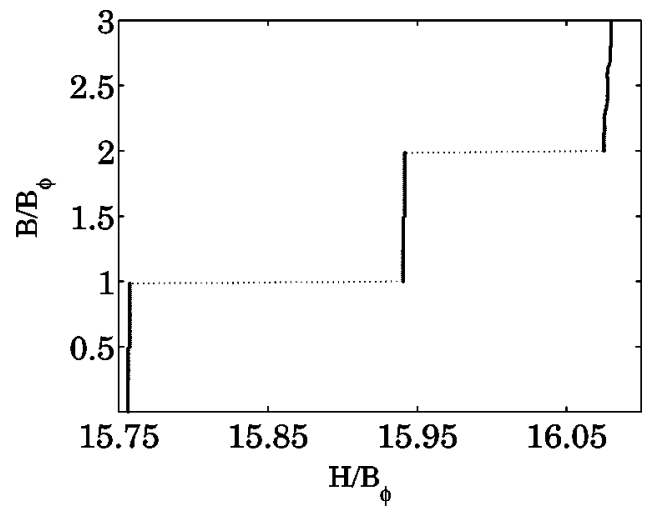

FIG. 15. The dependence of the magnetic induction $B$ on the applied field $H$ in the entire region of our simulation. A number of plateaus can be seen at points corresponding to simple rational filling fractions mentioned in the text. Only the vertical lines correspond to the data points obtained from our calculation; the dotted lines are guides to the eye. Both $B$ and $H$ have been scaled by the matching field $B_{\phi}$. 


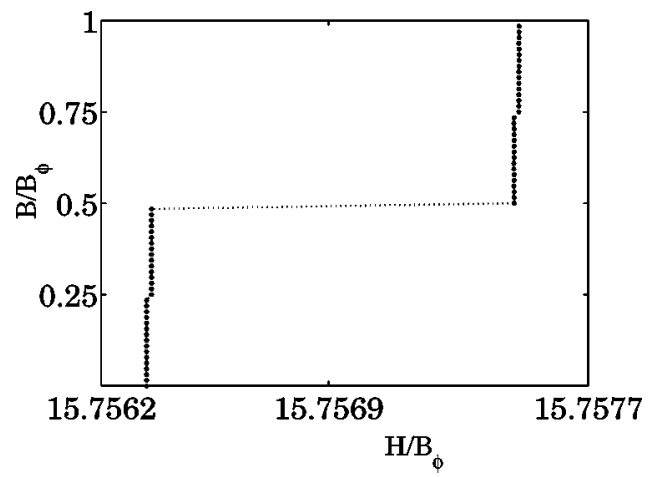

FIG. 16. Expanded view of the plot of Fig. 15 in the region between $n=B / B_{\phi}=0$ and $n=1$. One can see plateaus appearing at $n=1 / 4, n=1 / 2$, and $n=3 / 4$. The dark dots are the data points. The light dotted line is drawn to guide the eye.

shorter distance than those existing in the lattice or in its dual (i.e., the lattice obtained by replacing particles by holes, and vice versa). ${ }^{26}$ This makes sense because the introduction of this shorter distance introduces a larger energy scale, leading to a discontinuous change in the derivative of the energy with respect to the filling $n$. In the simulations, we have not scanned very small intervals of $n$. Also, for some of the fillings, the ground state may not have been obtained in our simulated annealing calculation. For these reasons, we cannot say anything definite about the true nature of the $B-H$ curve. It is possible that this curve has plateaus and discontinuities occurring at all scales (e.g., at all rational values of $n)$. The noisy nature of the $B-H$ plot in the range for which $n$ lies between 2 and 3 (see Fig. 17) is also due to these difficulties. However, this plot shows clear signatures of two plateaus that appear near $n=2.3$ and $n=2.6$. These can be understood to happen when first there is an occurrence of squares containing two vortices coming together next to each other diagonally [as in Fig. 3(a)], and again when they have to be next to each other with a common side [as in Figs. 3(b) and $3(\mathrm{c})$ ], as the value of $n$ increases from 2 to 3 .

\section{SUMMARY AND DISCUSSIONS}

In this paper, we have reported the results of an analytic calculation of the lowest-energy states of a vortex system in

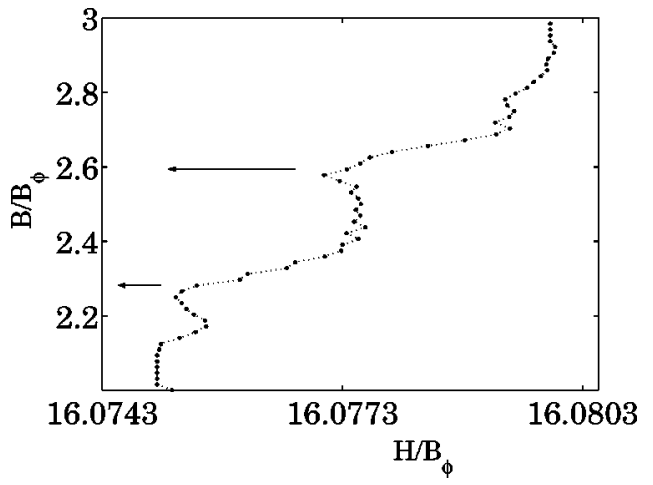

FIG. 17. Expanded view of the plot of Fig. 15 in the region between $n=2$ and $n=3$. One can see plateaus at fillings $n \simeq 2.3$ and $n \simeq 2.6$, as indicated by the arrows. The dark dots are the actual data points and the light dotted line is shown to guide the eye. the presence of a periodic array of strong pinning centers. We have considered several different lattice structures of the pin array and a large number of filling fractions in the range between zero and 4 . In our zero-temperature analysis, we have assumed that each pinning center traps a vortex if the number of vortices is less than or equal to the number of pinning centers, but a pinning center cannot trap more than one vortex. The analytic calculations are based on the principle of maximization of the shortest intervortex distance. We have argued that this principle leads to the exact ground states when the spacing of the defect lattice is large compared to the range of the intervortex interaction set by the value of the penetration depth (in our calculations, we assumed that the spacing of the pin lattice is ten times the penetration depth). This principle has been used, in combination with simple geometric considerations, to obtain the ground states for several values of the filling fraction $n$. The ground-state structures so obtained are found to be identical to those found in imaging experiments ${ }^{9}$ and in earlier simulations. ${ }^{17,20}$ We have also carried out simulated annealing calculations of the ground states in order to test some of the predictions of the analytic approach. In all cases, we found that the analytic results agree with those of our numerical calculations.

We have also described the results of a numerical calculation of the equilibrium magnetic induction $B$ and magnetization $M$ of a planar superconductor with a square array of pinning centers as functions of the externally applied field $H$. We show that the interplay between the lattice spacing of the pin array and the intervortex separation set by the value of $B$ leads to interesting commensurability effects, appearing as plateaus and discontinuities in the $B$ vs $H$ plot at simple rational values of the filling fraction $n$. Anomalies in the irreversible magnetization of thin-film superconductors with periodic arrays of pinning centers have been observed at certain integral values of $n$ in experiments ${ }^{3,4}$ and simulations. ${ }^{17}$ The presence of a periodic array of pins is also expected ${ }^{16}$ to produce anomalies in the equilibrium magnetization of the high-temperature vortex liquid at small integral values of $n$. Our results show that these commensurability effects are more pronounced in the field dependence of the equilibrium magnetization and magnetic induction of such systems in the low-temperature vortex-solid regime. Experimental investigations of these effects would be most welcome.

\section{APPENDIX: CONDITION FOR MAXIMIZING THE SHORTEST DISTANCE}

Given a particle fixed at some point in a plane, the problem is how to place three other particles around the first one in such a way that if we try to move the first particle from its position, it will get closer to at least one of the three particles. The solution is as follows. If we draw straight lines from the particle that we want to move to the other particles, then each angle between adjacent lines must be less than $180^{\circ}$. In other words, it should not be possible to draw a straight line through the particle in question in such a way that all the other three particles lie on one side of the line.

Let the particle that we want to move be at $P_{0}$ (see Fig. 


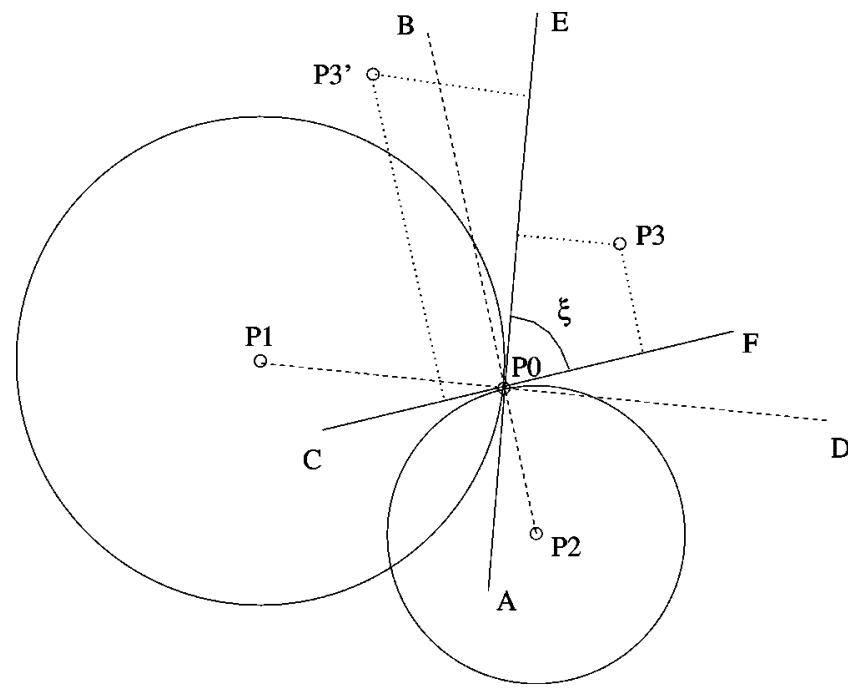

FIG. 18. Geometry of the problem of maximizing the shortest distance (see text).

18). Let us place two particles at $P_{1}$ and $P_{2}$, anywhere on the plane. This can be done since one of the angles between any two lines will always be less than or equal to $180^{\circ}$. In Fig. 18, $A E$ and $C F$ are the tangents to the circles centered at $P_{1}$ and $P_{2}$ and passing through point $P_{0}$. The presence of these particles restricts, to within the angle $\xi$, the direction in which the particle at $P_{0}$ can move without decreasing its distance to $P_{1}$ and $P_{2}$. Now let us check where we can fix the third particle such that the aforementioned condition is met, that is, the particle at $P_{0}$ cannot be moved without bringing it closer to one of the particles at $P_{1}, P_{2}$, and $P_{3}$. We will consider two possible cases - the third particle in the region $B P_{0} D$ of the plane and outside this region.

When the third particle is in the region $B P_{0} D$ (point $P_{3}$ in Fig. 18), it is not possible for particle $P_{0}$ to move from its position without decreasing the distance to any one of the particles. This can be seen if one drops the perpendiculars to the lines $A E$ and $C F$ from point $P_{3}$. If point $P_{0}$ is moved along either of these lines, it will be getting closer to point $P_{3}$. But that in turn implies that it cannot be moved into the region $\mathrm{FP}_{0} E$ at all without decreasing the distance from point $P_{3}$.

If the particle is outside the region $B P_{0} D$, for example, a point such as $P_{3}^{\prime}$, then it is easy to see that by moving along one of the tangents to the circles at point $P_{0}$, the particle at $P_{0}$ can move away from all three other points. Thus we find that the earlier statement we made is proved. This gives us a nice way to maximize the shortest distance since all one has to do is to make the three distances involved equal, so that if the central particle tries to move, then one of the distances has to decrease.
*Email address: toby@physics.iisc.ernet.in

†Email adddress: cdgupta@physics.iisc.ernet.in

${ }^{1}$ M. Tinkham, Introduction to Superconductivity (McGraw-Hill, New York, 1975).

${ }^{2}$ A. Abrikosov, Zh. Éksp. Teor. Fiz. 32, 1442 (1957) [Sov. Phys. JETP 5, 1174 (1957)].

${ }^{3}$ M. Baert, V.V. Metlushko, R. Jonckheere, V.V. Moshchalkov, and Y. Bruynseraede, Phys. Rev. Lett. 74, 3269 (1995).

${ }^{4}$ V.V. Moshchalkov, M. Baert, V.V. Metlushko, E. Rosseel, M.J. Van Bael, K. Temst, R. Jonckheere, and Y. Bruynseraede, Phys. Rev. B 54, 7385 (1996).

${ }^{5}$ A. Bezryadin and B. Pannetier, J. Low Temp. Phys. 102, 73 (1996).

${ }^{6}$ A.N. Grigorenko, G.D. Howells, S.J. Bending, J. Bekaert, M.J. Van Bael, L. Van Look, V.V. Moshchalkov, Y. Bruynseraede, G. Borghs, I.I. Kaya, and R.A. Strading, Phys. Rev. B 63, 052504 (2001).

${ }^{7}$ V. Metlushko, U. Welp, G.W. Crabtree, R. Osgood, S.D. Bader, L.E. DeLong, Z. Zhang, S.R.J. Brueck, B. Ilic, K. Chung, and P.J. Hesketh, Phys. Rev. B 60, R12 585 (1999).

${ }^{8}$ S.B. Field, S.S. James, J. Barentine, V. Metlushko, G. Crabtree, H. Shtrikman, B. Ilic, and S.R.J. Brueck, Phys. Rev. Lett. 88, 067003 (2002).

${ }^{9}$ K. Harada, O. Kamimura, H. Kasai, T. Matsuda, A. Tonomura, and V.V. Moshchalkov, Science 274, 1167 (1996).

${ }^{10}$ J.Y. Lin, M. Gurvitch, S.K. Tolpygo, A. Bourdillon, S.Y. Hou, and J.M. Phillips, Phys. Rev. B 54, R12 717 (1996).

${ }^{11}$ Jose I. Martin, M. Velez, J. Nogues, and I.K. Schuller, Phys. Rev. Lett. 79, 1929 (1997).
${ }^{12}$ D.J. Morgan and J.B. Ketterson, Phys. Rev. Lett. 80, 3614 (1998).

${ }^{13}$ Y. Jaccard, J.I. Martin, M.-C. Cyrille, M. Velez, J.L. Vincent, and I.K. Schuller, Phys. Rev. B 58, 8232 (1998).

${ }^{14}$ Jose I. Martin, M. Velez, A. Hoffmann, I.K. Schuller, and J.L. Vincent, Phys. Rev. Lett. 83, 1022 (1999).

${ }^{15}$ O.M. Stoll, M.I. Montero, J. Guimpel, J.J. Akerman, and I.K. Schuller, Phys. Rev. B 65, 104518 (2002).

${ }^{16}$ C. Dasgupta and D. Feinberg, Phys. Rev. B 57, 11730 (1998).

${ }^{17}$ C. Reichhardt, C.J. Olson, and F. Nori, Phys. Rev. B 57, 7937 (1998).

${ }^{18}$ C. Reichhardt and N. Gronbech-Jensen, Phys. Rev. B 63, 054510 (2001).

${ }^{19}$ C. Reichhardt, G.T. Zimanyi, and N. Gronbech-Jensen, Phys. Rev. B 64, 014501 (2001).

${ }^{20}$ L. Van Look, M.J. Van Bael, K. Temst, J.G. Rodrigo, M. Morelle, V.V. Moshchalkov, and Y. Bruynseraede, Physica C 332, 356 (2000).

${ }^{21}$ C. Dasgupta and O.T. Valls, Phys. Rev. Lett. 87, 257002 (2001); Phys. Rev. B 66, 064518 (2002).

${ }^{22}$ T. Joseph and C. Dasgupta, Phys. Rev. B 66, 212506 (2002).

${ }^{23}$ Q.-H. Wei, C. Bechinger, D. Rudhardt, and P. Leiderer, Phys. Rev. Lett. 81, 2606 (1998).

${ }^{24}$ E.R. Dufresne, G.C. Spalding, M.T. Dearling, S.A. Sheets, and D.G. Grier, Rev. Sci. Instrum. 72, 1810 (2001).

${ }^{25}$ G.S. Mkrtchyan and V.V. Shmidth, Zh. Éksp. Teor. Fiz. 61, 367 (1971) [Sov. Phys. JETP 34, 195 (1972)].

${ }^{26}$ G.I. Watson, Physica A 246, 253 (1997).

${ }^{27}$ T. Kennedy, Rev. Math. Phys. 6, 901 (1994). 